\title{
Impact of Coronavirus (COVID-19) on Otolaryngologic Surgery: A Brief Commentary
}

\author{
Darrin V. Bann ${ }^{1}$, Vijay A. Patel ${ }^{1}$, Robert Saadi ${ }^{1}$, John P. Gniady ${ }^{1}$, Neerav Goyal ${ }^{1}$, \\ Johnathan D. McGinn ${ }^{1}$, and David Goldenberg ${ }^{1}$ \\ ${ }^{1}$ Department of Otolaryngology - Head and Neck Surgery, The Pennsylvania State \\ University, College of Medicine, Hershey, PA USA
}

April 28, 2020

\begin{abstract}
Background: The Coronavirus disease - 2019 (COVID-19) pandemic is a global health crisis and Otolaryngologists are at increased occupational risk of contracting COVID-19. There are currently no uniform best-practice recommendations for Otolaryngologic surgery in the setting of COVID-19.

Methods: We reviewed relevant publications and position statements regarding the management of Otolaryngology patients in the setting of COVID-19. Recommendations regarding clinical practice during the Severe Acute Respiratory Syndrome (SARS) and Middle East Respiratory Syndrome (MERS) outbreaks were also reviewed.

Results: Enhanced personal protective equipment (N95 respirator and face shield or powered air-purifying respirator, disposable cap and gown, gloves) is required for any Otolaryngology patient with unknown, suspected, or positive COVID-19 status. Elective procedures should be postponed indefinitely, and clinical practice should be limited to patients with urgent or emergent needs.
\end{abstract}

Conclusion: We summarize current best-practice recommendations for Otolaryngologists to ensure safety for themselves, their clinical staff, and their patients.

\section{Introduction}

In December 2019, a pneumonia outbreak of unknown etiology was reported in Wuhan, Hubei province, China. ${ }^{1}$ This disease was subsequently named Coronavirus disease 2019 (COVID-2019) by the World Health Organization (WHO) and has spread globally affecting over 350,000 people and resulting in $>15,000$ deaths as of March 23, 2020. ${ }^{2}$ The causative agent was determined to be a novel Coronavirus (originally called 2019 novel Coronavirus or 2019-nCoV) closely related to Severe Acute Respiratory Syndrome (SARS) and Middle Eastern Respiratory Syndrome (MERS) viruses and has been named SARS-CoV-2. ${ }^{3}$ Although the full route of transmission is not clear, increasing evidence suggests that otolaryngologists are at increased risk of occupational SARS-CoV-2 exposure due to the high viral load in the upper aerodigestive tract and frequent examination and instrumentation of the oral cavity, oropharynx, nasal cavity, and nasopharynx. Indeed, the first physician deaths during the COVID-19 and SARS outbreaks were Otolaryngologists. ${ }^{4}$ In this commentary, we provide a brief overview of COVID-19 and provide recommendations for steps that Otolaryngologists should take to ensure the safety of themselves, operative teams, and patients. A summary of the recommendations discussed below is provided in Table 1. Given the rapidly evolving situation, these recommendations are based on the best available data and expert opinion, and may be subjected to change as the pandemic continues to develop.

Like all Coronaviruses, SARS-CoV-2 is an enveloped, positive-sense, single-stranded RNA virus. Sequence analysis has revealed $76.9 \%$ homology to SARS Coronavirus (SARS-CoV) and $96.4 \%$ homology to the bat Coronavirus BatCoVRaTG13 (RaTG13), indicating that SARS-CoV-2 is likely the result of a zoonotic 
infection. ${ }^{1}$ The viralspike $(S)$ protein interacts with the same cellular receptor, angiotensin-converting enzyme II (ACE2), as SARS-CoV, however, the SARS-CoV-2 $S$ protein has significant sequence divergence from all other known Coronaviruses except RaTG13. Importantly, SARS-CoV-2 appears to be highly transmissible with an $R_{0}$ of 2-3, meaning that each infected person is likely to infect 2-3 additional people in a naïve population. ${ }^{5}$ While respiratory droplet is presumed to be the major mode of transmission, evidence suggests that the virus may also be spread via the fecal-oral route and through the conjunctiva. ${ }^{6}$ Importantly, SARS-CoV-2 appears to be highly stable in the environment. Viable virus has been recovered from aerosols for up to 3 hours, porous surfaces (cardboard) for up to 24 hours, and non-porous surfaces (stainless steel, plastic) for up to 72 hours. ${ }^{7}$ This environmental stability increases the risk of nosocomial transmission and "super spreader" events, where a single individual infects more naïve individuals than expected by $R_{0} .^{7,8}$

The most commonly reported presenting symptoms of COVID-19 include cough (67.8\%), fever (43.8\%), fatigue $(38.1 \%)$, increased sputum production $(33.7 \%)$, dyspnea (18.7\%), myalgias $(14.9 \%)$, sore throat $(13.9 \%)$, and chills $(11.5 \%)^{9}$. However, some recent analyses indicate that hyposmia or anosmia and dysgeusia may be underrecognized symptoms. ${ }^{10,11}$ Laboratory testing may demonstrate leukopenia (33.7\%) and lymphopenia (83.2\%); elevated C-reactive protein $(60.7 \%)$, D-dimer $(46.4 \%)$, and lactate dehydrogenase (41\%) levels; transaminitis ( $20 \%)$; and decreased procalcitonin (5.5\%). Chest computed tomography $(\mathrm{CT})$ demonstrates abnormalities in $86.2 \%$ of cases including ground-glass opacities, patchy shadowing, and interstitial changes. ${ }^{9}$ SARS-CoV-2 appears to have a median incubation period of 5.1 days, with $95 \%$ of patients developing symptoms between 2.2 and 11.5 days after exposure. ${ }^{12}$ However, it is extremely important to recognize that asymptomatic patients may be highly contagious, ${ }^{12,13}$ highlighting the need for the use of personal protective equipment (PPE) for even routine patient evaluation.

\section{Infection Control Precautions}

In the setting of the current COVID-19 pandemic, routine head and neck examinations and head and neck procedures present a significant occupational hazard for physicians in the field of Otolaryngology. Instrumentation of the upper airway including the oral cavity, nose, naso/oro/hypopharynx, and larynx should be treated as high-risk procedures for transmission. Given this risk, otolaryngologic procedures should be deferred unless deemed medically necessary or until preoperative COVID-19 diagnostic testing is performed, particularly for Tier 1 and 2 patients, as defined by the American College of Surgeons. ${ }^{14,15}$ However, due to the acuity in workup and treatment of airway compromise, craniomaxillofacial trauma, and head and neck malignancy, examination and intervention will remain absolutely necessary in many patients during this time, without having COVID-19 testing in advance. Therefore, it is of utmost importance during diagnostic and therapeutic procedures that Otolaryngologists, as well as all other staff in the room, practice effective use of personal protective equipment. Perhaps the most easily implemented and effective method for limiting transmission is reducing redundant patient interaction overall, and minimizing the number of practitioners in the room at any time to perform examinations and procedures.

We define "appropriate PPE" as the use of standard-of-care procedure-specific PPE for patients who are confirmed to be negative for COVID-19 with appropriate pre-operative testing and quarantine and consideration for use of enhanced PPE in the appropriate setting. "Enhanced PPE" is defined as use of either an N95 respirator plus face shield or PAPR (preferred), disposable surgical cap, disposable gown, and gloves. This should be used for any patient with unknown, suspected, or positive COVID-19 status requiring invasive examination or instrumentation of the oral cavity, oropharynx, nasal cavity, or nasopharynx. The appropriate sequence of donning and removing PPE is also of importance (https://www.cdc.gov/hai/pdfs/ppe/PPESequence.pdf). ${ }^{16}$

There is conflicting data regarding mask protection for healthcare workers in the setting of pandemic airborne infections. Large meta-analyses have either demonstrated a lack of sufficient data to suggest superiority or no decreased risk with the use of N95 respirators compared to surgical masks in regard to laboratory-confirmed influenza. ${ }^{17,18}$ Regardless, N95 respirators are preferred in clinical settings when the risk of transmission is high given its superior filtration. However, the use of N95 respirators should be limited to healthcare workers who have been trained and properly mask fit tested. In general, face shields and surgical masks are 
mandatory during close patient encounters and may be used to cover the N95 respiratory masks if necessary. If the patient wears a mask for the entire encounter and the provider remains 3-6 feet away from the patient, a surgical mask may be sufficient, however this is not feasible for many otolaryngology clinical encounters. A suspected or known COVID-19 patient encounter requiring examination within 3 feet should proceed only with N95 respirator use, elastomeric respirator, or PAPR. ${ }^{19}$

With the current widespread shortage in supply of N95 respirators, the CDC has suggested using these respirators past their shelf life. Components of the respirators degrade over time; however, US stockpiles have been found to perform in accordance with the National Institute of Occupational Safety and Health (NIOSH) performance standards. ${ }^{19}$ Extended use may be preferable to limited reuse to decrease touching of the respirator, but both strategies are viable options. An exception to this is following an aerosol-generating procedure (i.e. tracheotomy, sinus surgery, oropharyngeal surgery, etc.), where it is recommended to discard the N95 respirator. Given the high viral load seen in the upper airway of COVID-19 infected patients, the use of a PAPR instead of an N95 respirator has been advocated. ${ }^{20-22}$ In a patient with unknown COVID-19 status requiring an upper airway procedure in the acute setting, enhanced PPE should be used even in the absence of suspicion for COVID-19 by history alone given that patients may be asymptomatic carriers or may be contagious prior to the development of symptoms. ${ }^{12,13}$ Consideration for excluding otolaryngologists who are of older age or have chronic medical conditions from interacting with COVID-19 patients should be made. During severe resource limitations when respirators are unavailable, convalescent doctors may be designated, although immunity following infection has not yet been confirmed and there is at least one report of disease recurrence in a convalescent patient. ${ }^{19,23}$

\section{Special Considerations}

\section{Airway Management and Tracheotomy}

Patients presenting with acute airway obstruction should be managed as if they are COVID-19 positive as diagnostic testing is not feasible in an emergent clinical situation. All clinical personnel should wear enhanced PPE. The use of high-flow nasal cannula is contraindicated in patients with unknown, suspected, or positive COVID-19 status due to high risk of virus aerosolization. ${ }^{24,25}$ Extreme caution should be utilized when performing awake fiberoptic intubation due to instrumentation of the nasopharynx and the potential for aerosol generation, however intubation via any means is preferable to emergent tracheotomy. A highefficiency hydrophobic filter should be placed between the face mask and breathing circuit or reservoir bag, the patient should be pre-oxygenation, and rapid sequence intubation techniques should be used to minimize viral particle aerosolization. ${ }^{25}$ When available, video laryngoscopes should be used to maximize intubation success rate and disposable laryngoscopes to minimize infectious spread. ${ }^{24,25}$

To minimize intubation time and exposure to the oropharynx, the 2015 Difficult Airway Society Guidelines ${ }^{26}$ should be followed with the exception that intubation should be performed only by the most senior practitioner available using enhanced PPE. Second-generation laryngeal mask airways should be used, if indicated, as these provide an improved seal compared to first-generation devices. ${ }^{25}$ If a "can't intubate, can't oxygenate scenario" is declared, emergent extra-corporeal membrane oxygenation (ECMO) may be preferred over emergent surgical airway to reduce the risk of virus aerosolization, though this may not be readily available. Indications for primary emergent tracheotomy include obstructive laryngeal lesions, severe trismus precluding the ability to perform direct laryngoscopy, massive oropharyngeal bleeding, other conditions precluding intubation, and other emergent conditions anticipated to require long-term means to secure the airway where ECMO would not be appropriate.

When caring for a patient with unknown, suspected, or positive COVID-19 status, clinical staff should be limited to essential personnel (i.e. senior attending anesthesiologist, experienced attending surgeon, senior surgical resident/clinical fellow, surgical technologist, and registered nurse) fully equipped with enhanced PPE in a negative pressure operating room with HEPA filtration ${ }^{24}$. Technical pearls to consider when performing tracheotomy in a patient with unknown, suspected, or positive COVID-19 status include avoiding electrocautery usage to minimize aerosolization of viral particles, advancing the endotracheal tube prior 
to incising the anterior tracheal wall to prevent cuff rupture and maintain a closed circuit, and holding ventilation until placement of cuffed non-fenestrated tracheotomy tube is confirmed with end-tidal CO2 and lung sound auscultation. Further details regarding safe tracheotomy have been outlined by Wei et al. and Harrison et al $.27,28$

Postoperatively, the tracheotomy tube should not be changed or manipulated until the COVID-19 status has been addressed with infectious disease. Routine tracheotomy tube care as delineated by ENT-UK should includes maintenance of a closed circuit, exclusively in-line suctioning, frequent cuff leak checks, and avoiding humidification. ${ }^{28}$ At this time elective tracheotomy is contraindicated for patients with unknown COVID-19 status and should only be performed once COVID-19 status has been determined with appropriate quarantine and the merits of tracheotomy are discussed as it is a high-risk, aerosol-generating procedure. ${ }^{29}$ Likewise, percutaneous dilation tracheotomy is contraindicated in patients with unknown, suspected, or positive COVID19 status. This contraindication is due to the need for simultaneous bronchoscopy (itself a high-risk procedure), and longer period of exposure to an open tracheostomy site during serial dilation resulting in increased risk of virus aerosolization.

There is limited information regarding management of tracheotomy patients no longer requiring ventilator support in the setting of COVID-19. The use of filters over the open tracheotomy, such as humidificationmoisture exchangers (HMEs) may be beneficial and reduce aerosolization. Alternatively, Chan et al. describe using a closed circuit system identical to that used for a mechanical ventilator for all tracheotomy patients, including those not requiring ventilator support. ${ }^{4}$ Ultimately, the choice of device may be dependent on the COVID-19 status of the individual patient and the ability to provide appropriate isolation to minimize the spread of aerosols.

\section{Endonasal Surgery}

At this time, several national and regional otolaryngology organizations have released statements regarding endonasal and nasopharyngeal surgery (functional endoscopic sinus surgery, endonasal skull base surgery, adenoidectomy, etc.) and the risk of COVID-19 spread. $^{30,31}$ In a preliminary study of 17 symptomatic COVID-19 patients, high viral loads were detected soon after symptom onset, with higher viral loads in the nasal cavity compared to the oropharynx. ${ }^{22}$ Increasing reports of hyposmia and anosmia as cardinal symptoms of CVOID-19 further suggest that the virus is highly active in the nasal cavity. ${ }^{11}$ Aerosolized viral particles are viable for up to 3 hours and may be disseminated by sinus instrumentation such as balloons, drills, microdebriders, and suction electrocautery. ${ }^{7,32}$ Elective sinonasal cases should be canceled for at least 1 month, and potentially longer. Urgent endonasal surgery cases mandate preoperative COVID-19 testing 48 hours prior to the procedure with the patient remaining in strict quarantine pending test results and appropriate PPE for all operating room personnel. Consideration should be given to postponing surgery for COVID-19 positive patients. Finally, emergent sinonasal cases require enhanced PPE for all operating room staff until further information is available ${ }^{32}$.

\section{Craniomaxillofacial Trauma and Urgent Otolaryngologic Conditions}

As emergency departments across the United States continue to serve patients with urgent medical needs, individuals who present with facial trauma, uncontrolled epistaxis, abscesses, and other conditions will require urgent management by otolaryngologists, including bedside procedures or operative intervention. Given our current understanding of COVID-19, the nasopharynx and nasal cavity harbor the highest viral load and protective precautions must be enforced with addressing injuries or urgent conditions in this location. We recommend the use of enhanced PPE for providers performing bedside procedures on patients with unknown, suspected, or positive COVID-19 status including repair of facial lacerations, management of animal bite wounds, control of epistaxis, peritonsillar abscess drainage, or any other condition requiring invasive examination or instrumentation of the oral cavity, oropharynx, nasal cavity, or nasopharynx. Enhanced PPE is indicated due to the increased risk aerosolization of saliva and nasal secretions in this setting. Patients presenting with operative facial fractures (e.g. orbital, mandibular, nasal, Le Fort pattern fractures, etc.), require preoperative COVID-19 diagnostic testing 48 hours prior to proceeding with elective surgical inter- 
vention with the patient kept in strict quarantine pending test results. In scenarios warranting emergent surgical intervention (e.g. rectus muscle entrapment, retrobulbar hemorrhage, flail mandible fractures, etc.) all operating room staff must utilize enhanced PPE as the COVID-19 status for these patients is unknown and should be presumed positive.

\section{Head and Neck Oncology}

At tertiary referral centers, many head and neck cancer patients have traditionally traveled far distances for oncologic care. In the setting of the COVID-19 pandemic, concerns exist for exposing patients to COVID19. Retroactive to January 27, 2020, the federal government has approved unprecedented steps to expand telemedicine services under Medicare and Medicaid with HIPAA flexibilities. ${ }^{33}$ This provides the ability to discuss pathology results and radiographic imaging findings with patients without direct patient contact, as well as continue important longitudinal cancer care. The more difficult decisions include delay of treatment for patients who are currently undergoing or starting chemoradiation. For solid tumor patients, adjuvant therapy with curative intent should proceed, despite the threat of COVID-19 infection during treatment. ${ }^{34}$ As outlined in the CMS Adult Elective Surgery and Procedures Recommendations, cancer surgery is categorized as a Tier 3a procedure and should not be postponed. ${ }^{15}$ Although oncologic procedures may continue as scheduled with preoperative COVID-19 diagnostic testing and quarantine, operative intervention requires prioritization; for example, definitive radiation therapy for a T1/T2 laryngeal carcinoma instead of a highrisk microscopic laryngeal resection using $\mathrm{CO} 2 / \mathrm{KTP}$ laser may be appropriate for some patients during the COVID-19 pandemic. Additional consideration may be given to patients undergoing resections requiring microvascular reconstruction due to anticipated postoperative hospitalization and use of hospital resources. Continued multidisciplinary discussions regarding all head and neck oncologic patients are essential. Similar to other otolaryngology procedures in suspected or confirmed patients with COVID-19, enhanced PPE should be used at all times as discussed above.

\section{Nasal Endoscopy and Flexible FIberoptic Laryngoscopy}

If the course of the COVID-19 pandemic is akin to the SARS outbreak, the impact on clinical activity at an academic otolaryngology department is expected to be substantial; over a 9-week period at the Prince of Wales Hospital in Hong Kong, the weekly outpatient clinic visits declined by $59 \%$, the number of operations performed dropped by $79 \%$, the average hospital capacity rate reduced by $79 \%$, and the daily admission rate diminished by $84 \% .{ }^{35}$ Furthermore, as viral density has been shown to be the highest in the nasal cavity and nasopharynx, elective diagnostics such as flexible laryngoscopy and nasal endoscopy should not be routinely performed in the office or inpatient setting. If endoscopic evaluation is required in the urgent or emergent setting, this should be only be performed using appropriate PPE and disposable nasal pledgets for decongestion and local anesthesia. A clinical study of 1,491 otolaryngology service consultations at Duke University from 2017 revealed 995 (66.7\%) of all consultations resulted in a bedside procedure and $243(16.3 \%)$ consultations required operative intervention. Consultations regarding airway evaluation (362 [47.3\%] vs 143 [19.7\%]) and rhinologic evaluation (79 [10.3\%] vs $18[2.5 \%])$ were more frequent from inpatient teams than from the emergency department. Of these evaluations, flexible fiberoptic laryngoscopy (554 [55.7\%]) and rigid nasal endoscopy (109 [11.0\%]) were two of the most commonly performed bedside procedures. In the operating room, direct laryngoscopy $(47[11.3 \%])$, bronchoscopy $(47[11.3 \%])$, and tracheotomy (41 [9.9\%]) were three of the most commonly performed operations. ${ }^{36}$ Continued demand for these critical services during the COVID-19 pandemic must be carefully evaluated by otolaryngologists on a case by case basis in light of CMS and AAO-HNS recommendations against elective endoscopy to mitigate risk of infectious spread and ensure patients who require such evaluations are carefully assessed using the proper precautions and appropriate PPE.

\section{Conclusion}

The COVID-19 pandemic has been deemed a global health emergency. Worldwide, the number of afflicted patients continues to rise and as of March 23, 2020 is placed at 354,677 cases with over 15,430 deaths. As members of the highest-risk surgical community, it is our responsibility to be aware of the aforementioned 
signs and symptoms and to promptly escalate suspected cases, particularly in those patients who require urgent or emergent otolaryngologic intervention. This discussion highlights important facts regarding COVID-19 for medical students, trainees, and surgeons and serves as a centralized resource for policymakers, health administrators, and hospital leadership as the medical field tirelessly combats this unprecedented viral outbreak.

\section{Table 1.}

\section{Hosted file}

hed-20-0403_table.docx available at https://authorea.com/users/5588/articles/438460-impact-ofcoronavirus-covid-19-on-otolaryngologic-surgery-a-brief-commentary

\section{References}

1. Zhou P, Yang XL, Wang XG, et al. A pneumonia outbreak associated with a new coronavirus of probable bat origin. Nature.2020;579(7798):270-273.

2. Johns Hopkins University \& Medicine. Coronavirus resource center. 2020; https://coronavirus.jhu.edu/. Accessed March 21, 2020.

3. Gorbalenya AE, Baker SC, Baric RS, et al. Severe acute respiratory syndrome-related coronavirus: the species and its viruses - a statement of the Coronavirus Study Group. bioRxiv. 2020. DOI: https://doi.org/10.1101/2020.02.07.937

4. Chan JYK, Wong EWY, Lam W. Practical Aspects of Otolaryngologic Clinical Services During the 2019 Novel Coronavirus Epidemic: An Experience in Hong Kong. JAMA Otolaryngol Head Neck Surg. 2020.

5. Liu Y, Gayle AA, Wilder-Smith A, Rocklov J. The reproductive number of COVID-19 is higher compared to SARS coronavirus. J Travel Med.2020;27(2).

6. Li JO, Lam DSC, Chen Y, Ting DSW. Novel Coronavirus disease 2019 (COVID-19): The importance of recognising possible early ocular manifestation and using protective eyewear. Br J Ophthalmol.2020;104(3):297298.

7. van Doremalen N, Bushmaker T, Morris DH, et al. Aerosol and Surface Stability of SARS-CoV-2 as Compared with SARS-CoV-1. N Engl J Med. 2020.

8. Liu Y, Eggo RM, Kucharski AJ. Secondary attack rate and superspreading events for SARS-CoV-2. Lancet.2020;395(10227):e47.

9. Guan WJ, Ni ZY, Hu Y, et al. Clinical Characteristics of Coronavirus Disease 2019 in China. $N$ Engl $J$ Med. 2020.

10. Mao L, Wang M, Chen S, et al. Neurological manifestations of hospitalized patients with COVID-19 in Wuhan, China: a retrospective case series. medRxiv. 2020. DOI: https://doi.org/10.1101/2020.02.22.20026500

11. Hopkins C, Kumar N. Loss of sense of smell as a marker of COVID-19 infection. 2020; https://www.entuk.org/losssense-smell-marker-covid-19-infection. Accessed March 21, 2020.

12. Lauer SA, Grantz KH, Bi Q, et al. The Incubation Period of Coronavirus Disease 2019 (COVID-19) From Publicly Reported Confirmed Cases: Estimation and Application. Ann Intern Med. 2020.

13. Bai Y, Yao L, Wei T, et al. Presumed Asymptomatic Carrier Transmission of COVID-19. JAMA. 2020.

14. American College of Surgeons. COVID-19: Guidance for triage of non-emergent surgical procedures. 2020; https://www.facs.org/about-acs/covid-19/information-for-surgeons/triage. Accessed March 21, 2020.

15. Siddiqui S. CMS adult elective surgery and procedures recommendations: limit all non-essential planned surgeries and procedures, including dental, until further notice. 2020; https://www.cms.gov/files/document/31820cms-adult-elective-surgery-and-procedures-recommendations.pdf. Accessed March 21, 2020. 
16. Centers for Disease Control and Prevention. Sequence for putting on personal protective equipment (PPE). 2020; https://www.cdc.gov/hai/pdfs/ppe/PPE-Sequence.pdf. Accessed March 21, 2020.

17. Smith JD, MacDougall CC, Johnstone J, Copes RA, Schwartz B, Garber GE. Effectiveness of N95 respirators versus surgical masks in protecting health care workers from acute respiratory infection: a systematic review and meta-analysis. CMAJ. 2016;188(8):567-574.

18. Long Y, Hu T, Liu L, et al. Effectiveness of N95 respirators versus surgical masks against influenza: A systematic review and meta-analysis.J Evid Based Med. 2020.

19. National Center for Immunization and Respiratory Diseases (NICIRD). Strategies for optimizing the supply of N95 respirators: crisis/alternate strategies. 2020; https://www.cdc.gov/coronavirus/2019-ncov/hcp/respiratorsstrategy/crisis-alternate-strategies.html. Accessed March 21, 2020.

20. Chee VW, Khoo ML, Lee SF, Lai YC, Chin NM. Infection control measures for operative procedures in severe acute respiratory syndrome-related patients. Anesthesiology. 2004;100(6):1394-1398.

21. Tien HC, Chughtai T, Jogeklar A, Cooper AB, Brenneman F. Elective and emergency surgery in patients with severe acute respiratory syndrome (SARS). Can J Surg. 2005;48(1):71-74.

22. Zou L, Ruan F, Huang M, et al. SARS-CoV-2 Viral Load in Upper Respiratory Specimens of Infected Patients. N Engl J Med.2020;382(12):1177-1179.

23. Chen D, Xu W, Lei Z, et al. Recurrence of positive SARS-CoV-2 RNA in COVID-19: A case report. Int J Infect Dis. 2020.

24. Kim HJ, Ko JS, Kim TY, Scientific Committee on Korean Society of A. Recommendations for Anesthesia in Patients Suspected of Coronavirus 2019-nCoV Infection. Korean J Anesthesiol. 2020.

25. Brewster DJ, Chrimes NC, Do TBT, et al. Consensus statement: safe airway society principles of airway management and tracheal intubation specific to the COVID-19 adult patient group. The Medical Journal of Australia. 2020;E-pub ahead of print.

26. Frerk C, Mitchell VS, McNarry AF, et al. Difficult Airway Society 2015 guidelines for management of unanticipated difficult intubation in adults. Br J Anaesth. 2015;115(6):827-848.

27. Wei WI, Tuen HH, Ng RW, Lam LK. Safe tracheostomy for patients with severe acute respiratory syndrome. Laryngoscope.2003;113(10):1777-1779.

28. Harrison L, Ramsden J, Winter S, Rocke J, Heward E. Guidance for surgical tracheostomy and tracheostomy tube change during the COVID-19 pandemic. 2020; https://www.entuk.org/tracheostomy-guidanceduring-covid-19-pandemic. Accessed March 21, 2020.

29. The Faculty of Intensive Care Medicine, Intensive Care Society, Association of Anaesthetists, and Royal College of Anaesthetists. Information, guidance and resources supporting the understanding and management of Coronavirus (COVID-19). 2020; https://icmanaesthesiacovid-19.org/. Accessed March 21, 2020.

30. American Rhinological Society. Coronavirus (COVID-19) updates. 2020; https://www.american-rhinologic.org/. Accessed March 21, 2020.

31. American Academy of Otolaryngology - Head \& Neck Surgery. Coronavirus Disease 2019: Resources. 2020; https://www.entnet.org/content/coronavirus-disease-2019-resources. Accessed March 21, 2020.

32. Patel ZM, Fernandez-Miranda J, Hwang PH, et al. Precautions for endoscopic transnasal skull base surgery during the COVID-19 pandemic.Neurosurgery. 2020;E-pub ahead of print.

33. Hildrew DM, Committee A-HT. Prioritizing novel approaches to telehealth for all practitioners. 2020; https://www.entnet.org/content/prioritizing-novel-approaches-telehealth-all-practitioners. Accessed March $21,2020$. 
34. Ueda M, Martins R, Hendrie PC, et al. Managing Cancer Care During the COVID-19 Pandemic: Agility and Collaboration Toward a Common Goal.J Natl Compr Canc Netw. 2020:1-4.

35. Vlantis AC, Tsang RK, Wong DK, Woo JK, van Hasselt CA. The impact of severe acute respiratory syndrome on otorhinolaryngological services at the Prince of Wales Hospital in Hong Kong. Laryngoscope.2004;114(1):171-174.

36. Choi KJ, Kahmke RR, Crowson MG, Puscas L, Scher RL, Cohen SM. Trends in Otolaryngology Consultation Patterns at an Academic Quaternary Care Center. JAMA Otolaryngol Head Neck Surg. 2017;143(5):472477 . 